\title{
You-Do, I-Learn: Discovering Task Relevant Objects and their Modes of Interaction from Multi-User Egocentric Video
}

\section{Dima Damen}

Dima.Damen@bristol.ac.uk

Teesid Leelasawassuk

Csztl@bristol.ac.uk

Osian Haines

Osian.Haines@bristol.ac.uk

Andrew Calway

Andrew.Calway@bristol.ac.uk

Walterio Mayol-Cuevas

Walterio.Mayol-Cuevas@bristol.ac.uk

\author{
Computer Science Department \\ University of Bristol \\ Bristol, UK
}

We present a fully unsupervised approach for the discovery of i) task relevant objects and ii) how these objects have been used. A Task Relevant Object (TRO) is an object, or part of an object, with which a person interacts during task performance. Given egocentric video from multiple operators, the approach can discover objects with which the users interact, both static objects such as a coffee machine as well as movable ones such as a cup. Importantly, we also introduce the term Mode of Interaction (MOI) to refer to the different ways in which TROs are used. Say, a cup can be lifted, washed, or poured into. When harvesting interactions with the same object from multiple operators, common MOIs can be found. Setup and Dataset: Using a wearable camera and gaze tracker (Mobile Eye-XG from ASL), egocentric video is collected of users performing tasks, along with their gaze in pixel coordinates. Six locations were chosen: kitchen, workspace, laser printer, corridor with a locked door, cardiac gym and weight-lifting machine. The Bristol Egocentric Object Interactions Dataset is publically available ${ }^{1}$.

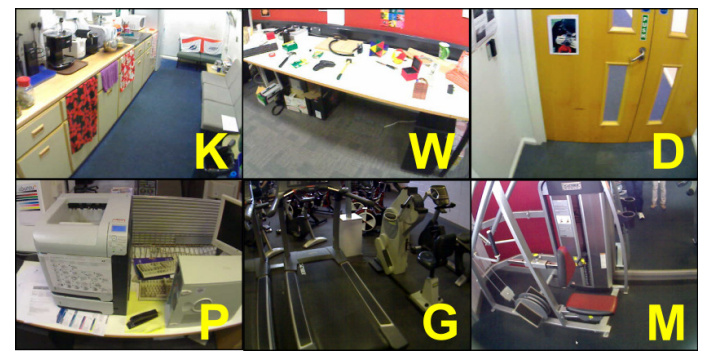

Figure 1: An overview of the locations in the dataset.

Discovering TROs: Given a sequence of images $\left\{I_{1}, . ., I_{T}\right\}$ collected from multiple operators around a common environment, we aim to extract $K$ TROs, where each object $T R O_{k}$ is represented by the images from the sequence that feature the object of interest . We investigate using appearance, position and attention, and present results using each and a combination of relevant features. For attention, we exploit the high quality and predictive nature of eye gaze fixations.

Results compare k-means clustering to spectral clustering, and propose estimating the optimal number of clusters using the standard DaviesBouldin (DB) index. Figure 2 shows the best performance for discovering TROs by combining position (relative to a map of the scene) and appearance (HOG features within BoW) over a sliding window $w=25$, using gaze fixations for attention, spectral clustering and estimating the number of clusters using the Davies-Bouldin (DB) index.

Finding MOIs: Given consecutive images $\left(I_{t}, I_{t+1}, I_{t+\rho}\right)$ clustered into the same TRO, a video snippet $u_{i}^{k}$ for TRO $k$ is defined as

$$
u_{i}^{k}=\left\{\Psi\left(I_{j}, \Delta(j), \omega\right) ; \quad I_{j} \in T R O_{k} ; \quad j=t . . t+\rho ; \quad \rho \geq \xi\right\}
$$

where $\Psi$ crops a window of size $\omega$ from image $I_{j}$ around $\Delta(j)$, and $\Delta(j)$ is the interpolated gaze at frame $j$ as gaze information is missing in some frames. The collection of all video snippets $U_{k}=\left\{u_{i}^{k}\right\}$ shows different ways in which $T R O_{k}$ was used.

On average, 16.6 video snippets are extracted for each TRO $(\sigma=7.4)$. We cluster $U_{j}$, and represent each cluster by the video snippet $\hat{u}_{j}$ closest to the centre of the cluster $\mu_{j}$ (i.e. mean snippet), as well as the percentage of

${ }^{1}$ http://www.cs.bris.ac.uk/ damen/BEOID/

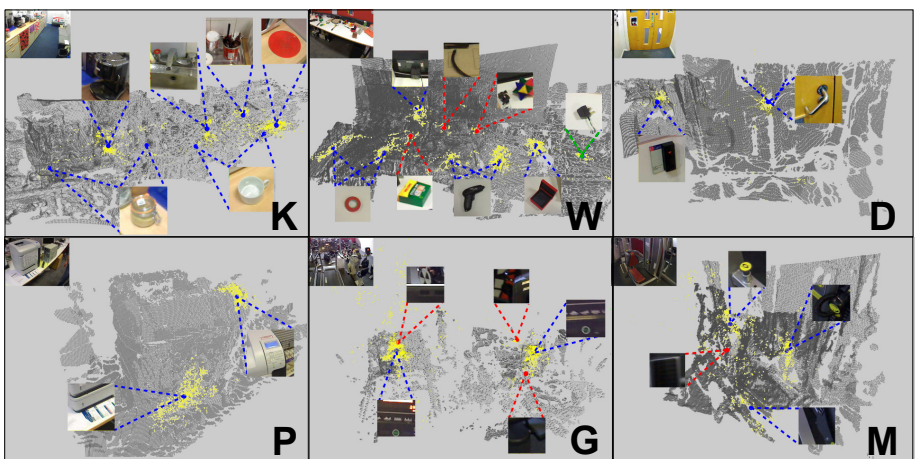

Figure 2: Discovered TROs. An overview of the locations is shown at the top. Blue dots represent true-positive (19 objs), red dots represent false positive ( $7 \mathrm{objs}$ ) and green dots represent false negative ( $1 \mathrm{obj})$.

snippets within that cluster $p\left(M O I_{j}\right)$. We vary the threshold $\lambda$ to accept $p\left(M O I_{j}\right)$ to produce recall-precision curves. Figure 3 shows an example of the method successfully discovering two MOIs for the 'socket'.

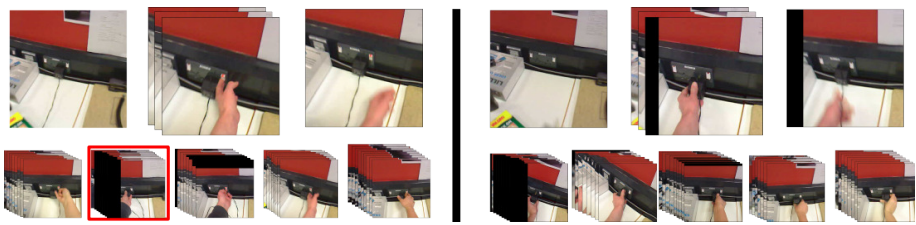

Figure 3: For the 'socket', the two common MOIs ('switching', 'plugging') are found (left \& right). The representative video snippet is shown (up) with the other snippets in the same cluster (below) - only one snippet is incorrectly clustered (shown in red).

Video Guides: In addition, the approach enables the automatic generation of help snippets on how objects have been used before. We showcase video help guides using inserts on a pre-recorded video. A suitable video insert (i.e. MOI snippet) is chosen every time a gazed-at object is first recognised. In this assistive mode, we use the real-time texture-minimal scalable detector ${ }^{2}$ due to its light-weight computational load that makes it amendable to wearable systems. Figure 4 shows frames from the help videos and a full sequence is available ${ }^{3}$. Recall that these inserts are extracted, selected and displayed fully automatically.
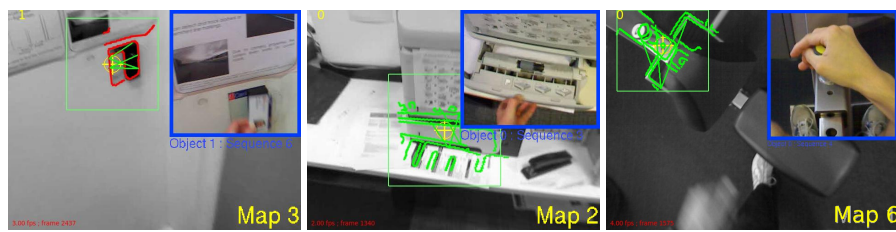

Figure 4: In the assistive mode, when a TRO is detected, video snippet is inserted showing the most relevant common MOI based on the object's current appearance.

${ }^{2}$ http://www.cs.bris.ac.uk/ damen/MultiobjDetector.htm

${ }^{3}$ http: //www,cs,bris,ac,uk/ damen/You-Do-I-Learn 